\section{N. Guo*, F. Marra*,\#, J.M. FitzGerald", R.K. Elwood ${ }^{\#, \oplus}$ and C.A. Marra*}

*Faculty of Pharmaceutical Sciences, University of British Columbia, "British Columbia Centre for Disease Control, and "Faculty of Medicine, University of British Columbia, Vancouver, BC, Canada.

Correspondence: C.A. Marra, Faculty of Pharmaceutical Sciences, University of British Columbia, 2146 East Mall, V6T $1 Z 3$ Vancouver, BC, Canada. E-mail: carlo.marra@ubc.ca

Statement of Interest: None declared.

\section{REFERENCES}

1 Forget EJ, Menzies D. Adverse reactions to first-line antituberculosis drugs. Expert Opin Drug Saf 2006; 5: 231-249.
2 Marra F, Marra CA, Bruchet $\mathrm{N}$, et al. Adverse drug reactions associated with first-line anti-tuberculosis drug regimens. Int $J$ Tuberc Lung Dis 2007; 11: 868-875.

3 Guyatt GH, Feeny DH, Patrich DL. Measuring health-related quality of life. Ann Intern Med 1993; 118: 622-629.

4 Ware JE Jr, Kosinski M, Bjorner JB, et al. User's manual for the SF36v2 ${ }^{\circledR}$ Health Survey. $2^{\text {nd }}$ edn. Lincoln, RI: Quality Metric Incorporated, 2007.

5 Gold M, Franks P, Erickson P. Assessing the health of the nation. The predictive validity of a preference-based measure and self-rated health. Med Care 1996; 34: 163-177.

6 Franks P, Gold MR, Fiscella K. Sociodemographics, self-rated health, and mortality in the US. Soc Sci Med 2003; 56: 2505-2514.

7 Sehlen S, Lenk M, Hollenhorst H, et al. Quality of Life (QoL) as Predictive Mediator Variable for Survival in Patients with Intracerebral Neoplasma During Radiotherapy. Onkologie 2003; 26: 38-43.

\title{
Development of a standardised tool to survey MDR-/XDR-TB case management in Europe
}

\section{To the Editors:}

The emergence of multidrug-resistant tuberculosis (MDR-TB, defined as in vitro resistance to isoniazid and rifampicin) [1-5] and extensively drug-resistant TB (XDR-TB, defined as in vitro drug resistance to isoniazid and rifampicin plus any fluoroquinolone and at least one of the injectable drugs: capreomycin, kanamycin or amikacin) represents a major threat to TB control at the global level [1-5].

In 2007, the World Health Organization (WHO) estimated a prevalence of 511,000 MDR-TB cases (with 150,000 deaths) and 50,000 XDR-TB cases (and 30,000 deaths) [1]; 14 out of 19 highMDR-TB burden territories are located in Former Soviet Union (FSU) countries [1].

$\mathrm{XDR}-\mathrm{TB}$ is a manmade product, resulting, in essence, from clinical mismanagement of newly diagnosed pan-susceptible TB cases as well as MDR-TB cases [1,2]. There is evidence that suboptimal TB case management in parts of Europe contributes to the development of resistance to the XDR-TB defining drugs [4-7].

In spite of the growing amount of public awareness about TB drug resistance, the essential variables necessary to fully understand MDR-TB and XDR-TB are unfortunately not systematically collected, analysed and reported in published studies [2, 8]. Although the international community is providing a rapid response to XDR-TB (formulation of an emergency plan [9]; development of an instrument to support implementation and scale-up of national strategies and to assess programmatic needs [10]), no standardised and comprehensive tool is available to survey the key factor responsible for the emergence of MDR-/ XDR-TB: inappropriate TB case management.
The aim of this letter is to describe the process undertaken by the Tuberculosis Network European Trialsgroup (TBNET) in collaboration with the European Centre for Disease Prevention and Control (ECDC) to develop a standardised tool to survey MDR-/XDR-TB case management in the European Union/ European Economic Area (EU/EEA) countries and the validation process used to finalise the tool.

This instrument was devised to survey original clinical records of susceptible TB and MDR-/XDR-TB cases to ascertain if case management activities were performed according to established guidelines and national regulations [11, 12]. It was not designed to detect pathogenetic mechanisms or risk factors involved in the emergence of drug-resistance in selected TB cases.

The tool identifies the most critical gaps in susceptible TB/ MDR-/XDR-TB case management that need to be addressed urgently to prevent adverse outcomes for both individual patient and public health.

Data elements were organised taking into account the following. 1) Structure and content of clinical records in a sample of European countries participating in the ECDC European survey on case management of MDR-TB (five EU countries, representing different $\mathrm{TB}$ and MDR-TB incidence: one high TB/high MDR-TB; two low TB/low MDR-TB in Northern and Southern Europe; one high TB/intermediate MDR-TB; and one intermediate TB/low MDR-TB). 2) The recommendations of the TBNET systematic review on XDR-TB management [2]: a) prospective study design, standardised, internationally accepted definitions, quality-controlled laboratory testing for all first- and second-line drugs defining XDRTB; agreed-upon set of standard variables allowing for comparison of approaches and results across studies; b) the 
variables collected should include: measures of disease severity (number of drugs to which isolates are resistant, clinical features), treatment history, number and type of active drugs used in treatment, time to sputum smear and culture conversion, treatment duration, adverse events related to treatment and treatment outcome.

The tool is composed of three parts and one annex: Part 1 summarises the features of the setting surveyed (62 items); Part 2 collects information on individual clinical records surveyed (one line per clinical case, 141 items); Part 3 allows comparison of the key case management decisions taken on each individual case against internationally agreed standards (25 scored questions). Standards are summarised in the Annex for easy reference.

Development of the draft tool included item formulation and two reviews by a core group of experts in specific areas of TB control and one review of the draft tool by a larger international expert group. Two phases of pre-testing occurred, including use by writing committee members, and desk simulations using data from clinical records of previous TBNET studies [4-7]. A preliminary field test was conducted at Sondalo Hospital (Italy) in October 2009 (19 records). The final revision and presentation of the tool took place at an initial workshop (Stockholm, 19 November 2009), where both consultants and clinical counterparts from each clinical site to be surveyed were trained in its use.

During testing, data elements were added, consolidated, revised or deleted based on their utility in evaluating the key elements of MDR/XDR-TB case management. The tool incorporates a scoring system for the final survey data elements. The scoring system was designed to identify areas for strengthening within the MDR-/XDR-TB case management by a self-evaluation. Scores are summed by survey component (medical history, diagnosis, treatment, treatment monitoring, outcome allocation, infection control) only, i.e. no overall score is produced, and provide a relative measure of the strengths and gaps between components to help prioritise remedial actions.

Scores were assigned through a Delphi process aimed at defining a specific weight for each data element in terms of importance for MDR/XDR-TB case management based on expert opinion. As no data exist to rank the essential elements of MDR-/XDR-TB case management, 35 expert reviewers were asked to assign a ranking score to each element (from highest to lowest importance on a scale of 1-5). Data element scores were then weighted according to the mean value assigned by the expert panel. Each component in Part 3 is followed by summary scores and a proposed interpretation of score ranges (see table 1).

From the first to the final draft, the total number of columns increased from 187 to 228 (for Part 1 from 55 to 62, for Part 2 from 108 to 141, for Part 3 from 24 to 25). In pre-testing and pilot evaluations, the tool proved useful in collecting the relevant data for evaluation of case management and provided data that can be used to improve clinical practices.

The simulation using Sondalo Hospital (Italy) data identified two "major" deviations from internationally agreed standards

\section{TABLE 1}

Proposed interpretation of score ranges for each element of the case management survey tool

Question weight Score

\section{Component 1: Medical history}

Investigation previous TB diagnosis

Investigation previous DST

Investigation previous treatment

Investigation contacts

$4.5 \pm 0.7$

$4.6 \pm 0.6$

$3.3 \pm 1.1$

Section score (maximum 67); possible interpretation

$>53$; no major gaps

53-35; some critical gaps needing action

$<35$; major gaps

\section{Component 2: Diagnosis}

Diagnostic algorithm

Microbiology

Other examinations

Final decision

$4.1 \pm 1$

$4.9 \pm 0.4$

$3.5 \pm 0.9$

$4.6 \pm 0.9$

Section score (maximum 68); possible interpretation

$>53$; no major gaps

53-38; some critical gaps needing action

$<38$; major gaps

\section{Component 3: Treatment}

TB regimen choice (four active drugs ensured)

$4.9 \pm 0.4$

$4.9 \pm 0.4$

Dosage

Duration

$4.5 \pm 0.8$

$3.7 \pm 1$
$3.5 \pm 1.1$

HIV regimen choice

Management adverse events HIV treatment

Section score (maximum 105); possible interpretation

>91; no major gaps

91-76; some critical gaps requiring action

$<76$; major gaps

\section{Component 4: Treatment monitoring}

Necessary examinations performed

Section score (maximum 18); possible interpretation

18; no major gaps

0; major gaps

\section{Component 5: Outcome}

Correct outcome assigned

$4.7 \pm 0.5$

Section score (maximum 19); possible interpretation

19; no major gaps

0; major gaps

\section{Component 6: Infection control}

Administrative measures adequate

Environmental measures adequate

Infection control committee

Surveillance system for drug-resistance in the setting

Staff personal protective measures adequate

Cough etiquette

Availability of respirators

Training of the staff on infection control

$4.5 \pm 0.7$

Section score (maximum: 129); possible interpretation

$>114$; no major gaps

114-99; some critical gaps requiring action

$<99$; major gaps

Data are presented as score or mean \pm SD. TB: tuberculosis; DST: drug susceptibility testing; Question mean weight: value assigned by expert opinion to the given question in a scale from 1 to 5; Score: actual value of each question composing case management survey tool, at the end of each section of the tool a guide for the possible interpretation of the score achieved by managing each individual case is given. The Section score is designed to guide interpretation of the score sum obtained for every item/question included in Part 3 of the tool. In case of a positive assessment (the activity was managed according to the established guidelines) the maximum score will be given. Zero is assigned in case of a negative assessment. 
in the area of infection control (no negative pressure ventilation available, no respirator fit testing done) and the impossibility to perform all second-line drug susceptibility testing (DST) at the Reference Centre (capreomycin), as well as three "major" deviations related to drug choice and two "critical" deviations related to the diagnostic algorithm in hospitals managing MDR-TB cases before referring them to a Reference Centre (see definitions in table 1).

Analysis of risk factors associated to drug resistance in individual patients is not allowed by the present tool, so that health indicators like odds ratios or relative risks will not be calculated.

As mentioned above, the tool was designed to ascertain if case management activities were performed according to established guidelines. If, for example, the clinician has investigated the patient's previous treatment history or previous DST, he has followed the guidelines even if the information obtained is not complete (as often happens in real life).

As this tool was planned to perform a meticulous survey of the original clinical records of $40 \mathrm{~TB} / \mathrm{MDR}-\mathrm{TB}$ cases (as recommended by international guidelines [13]) in one national reference centre in five different EU member states, the time needed to complete the exercise was relevant: it ranged from 4 to 5 days for a team of two to three trained auditors supported by trained local clinicians.

In conclusion, a standardised tool to survey susceptible TB and MDR-/XDR-TB case management will support global efforts to prevent and contain MDR-/XDR-TB by identifying pitfalls that could benefit from programmatic intervention. The present tool proved able to differentiate two pitfalls in case management occurring in national reference centres and five in general hospitals before cases were referred.

The next steps of the study include surveying the participating European countries and development of a comprehensive report to inform on further actions needed to prevent occurrence of MDR-/XDR-TB in the European Union from a public health perspective.

\section{G. Sotgiu*, R. Centis", L. D'Ambrosio", S. De Lorenzo", M. D'Arcy Richardson ${ }^{+}$, C. Lange ${ }^{\S}$, D. Manissero ${ }^{f}$ and G.B. Migliori", for the TBNET MDR-TB project**}

${ }^{*}$ Hygiene and Preventive Medicine Institute, University of Sassari, Sassari, "WHO Collaborating Centre for TB and Lung Diseases, Fondazione S. Maugeri, Care and Research Institute, Tradate, `E. Morelli Hospital, Sondalo, Italy. ${ }^{+} \mathrm{HIV} / \mathrm{TB}$ Global Program, PATH, Seattle, Washington, USA. ${ }^{\S}$ Division of Clinical Infectious Diseases, Medical Clinic, Research Centre Borstel, Borstel, Germany. ${ }^{f}$ Scientific Advice Unit, European Centre for Disease Prevention and Control, Tomtebodavägen, Stockholm, Sweden. ${ }^{* *}$ A full list of the TBNET MDR-TB project members and their affiliations can be found in the Acknowledgements section.

Correspondence: G.B. Migliori, WHO Collaborating Centre for $\mathrm{TB}$ and Lung Diseases, Fondazione S. Maugeri, Care and Research Institute/TBNET Secretariat (TuBerculosis Network
European Trialsgroup)/Stop TB Italy, via Roncaccio 16, 21049, Tradate, Italy. E-mail: giovannibattista.migliori@fsm.it

Support Statement: TBNET performed the study under the ECDC service contract 1757.

Statement of Interest: None declared.

Acknowledgements: The members of the TBNET MDR-TB Project are as follows. K. Kliiman (University of Tartu, Tartu, Estonia); G. Guenther (Research Center Borstel, Borstel, Germany); R. Muetterlein (Parsberg Hospital, Parsberg, Germany); M. Sester (University of the Saarland, Homburg, Germany); D. Wagner (University Medical Center Freiburg, Freiburg, Germany); D. Cirillo (San Raffaele Scientific Institute, Milan, Italy); D. Goletti (National Institute for Infectious Diseases L. Spallanzani, Rome, Italy); M. Villar (Public Health Office, Lisbon, Portugal); V. Spanu (Marius Nasta Institute of Pulmonology, Bucharest, Romania); J-P. Zellweger (Swiss Lung Association, Bern, Switzerland); G. Bothamley (Homerton University Hospital, London, UK); and B. Kampmann (Imperial College London, London, UK).

Following is a list of experts who provided input for the Delphi Process. A. Altraja and K. Kliiman (University of Tartu, Tartu, Estonia); G. Guenther and C. Lange (Research Center Borstel, Borstel, Germany); D. Wagner (University Medical Center Freiburg, Freiburg, Germany); F. Blasi (University of Milan, Milan, Italy); G.B. Migliori, R. Centis, L. D'Ambrosio and A. Facchini (S. Maugeri Foundation, Tradate, Italy); G. Besozzi and S. De Lorenzo (E. Morelli Hospital, Sondalo, Italy); G. Ferrara and L. Casali (University of Perugia, Perugia, Italy); D. Goletti (National Institute for Infectious Diseases L. Spallanzani, Rome, Italy); A. Gori (University Milan-Bicocca, Milan, Italy); A. Matteelli and A.C. Carvalho (University of Brescia, Brescia, Italy); A. Scardigli (Columbia University, Maputo, Mozambique); G. Sotgiu, P. Castgilia and A. Piana (University of Sassari, Sassari, Italy); A. Spanevello (UniversitÁ degli Studi dell'Insubria, Varese, Italy); M. Tadolini (World Health Organization Consultant, Bologna, Italy); M. Villar (Public Health Office, Lisbon, Portugal); L. Ditiu (World Health Organization, Geneva, Switzerland), E. Ibraim, V. Spanu and D. Chiotan (Marius Nasta Institute of Pulmonology, Bucharest, Romania); O. Toungoussova (Northern State Medical University, Archangels, Russian Federation); D. Manissero (European Centre for Disease Prevention and Control, Stockholm, Sweden); M. Fuad (World Health Organization, Geneva, Switzerland); J-P. Zellweger (Swiss Lung Association, Berne, Switzerland); G. Bothamley (Homerton University Hospital, London, UK); B. Kampmann (Imperial College London, Lonon, UK); and M. D'Arcy Richardson (PATH, Seattle, USA).

\section{REFERENCES}

1 World Health Organization. Anti-tuberculosis drug resistance in the world. Fourth global report. WHO/HTM/TB/2008.394. Geneva, World Health Organization, 2008.

2 Sotgiu G, Ferrara G, Matteelli A, et al. Epidemiology and clinical management of XDR-TB: a systematic review by TBNET. Eur Respir J 2009; 33: 871-881.

3 Migliori GB, Loddenkemper R, Blasi F, et al. 125 years after Robert Koch's discovery of the tubercle bacillus: the new XDR-TB threat. Is "science" enough to tackle the epidemic? Eur Respir J 2007; 29: 423-427.

4 Migliori GB, Besozzi G, Girardi E, et al. Clinical and operational value of the extensively drug-resistant tuberculosis definition. Eur Respir J 2007; 30: 623-626.

5 Migliori GB, Ortmann J, Girardi E, et al. Extensively drug-resistant tuberculosis, Italy and Germany. Emerg Infect Dis. 2007; 13: 780-782.

6 Migliori GB, Lange C, Centis R, et al. Resistance to second-line injectables and treatment outcomes in multidrug-resistant and 
extensively drug-resistant tuberculosis cases. Eur Respir J 2008; 31: 1155-1159.

7 Migliori GB, Lange C, Girardi E, et al. Fluoroquinolones: are they essential to treat multidrug-resistant tuberculosis? Eur Respir J 2008; 31: 904-905.

8 Migliori GB, Richardson MD, Lange C. Of blind men and elephants: making sense of extensively drug-resistant tuberculosis. Am J Respir Crit Care Med. 2008; 178: 1000-1001.

9 World Health Organization. Extensively drug-resistant tuberculosis (XDR-TB): recommendations for prevention and control. Wkly Epidemiol Rec 2006; 81: 430-432.

10 Migliori GB, Sotgiu G, Jaramillo E, et al. Development of a standardised multidrug-resistant/extensively drug-resistant tuberculosis assessment and monitoring tool. Int J Tuberc Lung Dis 2009; 13: 1305-1308.
11 Migliori GB, Hopewell PC, Blasi F, et al. Improving the TB case management: the International Standards for Tuberculosis Care. Eur Respir J 2006; 28: 687-690.

12 World Health Organization. Guidelines for the programmatic management of drug-resistant tuberculosis WHO/HTM/TB/ 2008.402. Geneva, WHO, 2008.

13 Broekmans JF, Migliori GB, Rieder HL, et al. European framework for tuberculosis control and elimination in countries with a low incidence. Recommendations of the World Health Organization (WHO), International Union against Tuberculosis and Lung Disease (IUATLD) and Royal Netherlands Tuberculosis Association (KNCV) Working Group. Eur Respir J 2002; 19 765-775.

DOI: $10.1183 / 09031936.00186409$ 